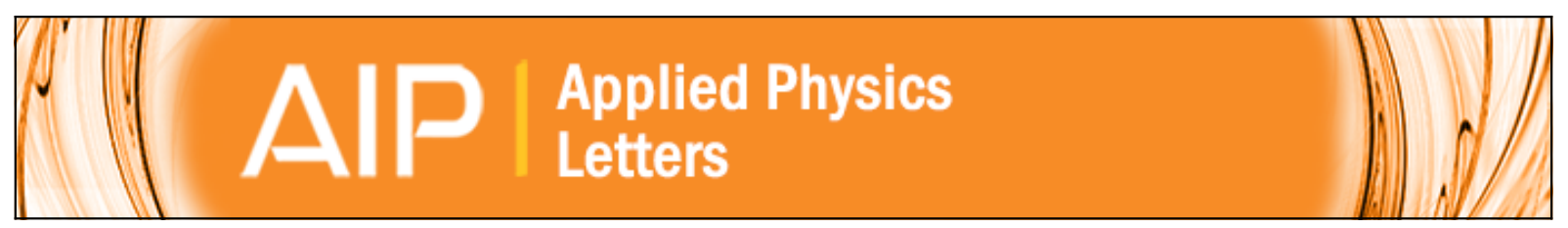

\title{
Low dimensional GaAs/air vertical microcavity lasers
}

J. Gessler, T. Steinl, A. Mika, J. Fischer, G. Sk, J. Misiewicz, S. Höfling, C. Schneider, and M. Kamp

Citation: Applied Physics Letters 104, 081113 (2014); doi: 10.1063/1.4866805

View online: http://dx.doi.org/10.1063/1.4866805

View Table of Contents: http://scitation.aip.org/content/aip/journal/apl/104/8?ver=pdfcov

Published by the AIP Publishing

\section{Articles you may be interested in}

High reflectance membrane-based distributed Bragg reflectors for GaN photonics

Appl. Phys. Lett. 101, 221104 (2012); 10.1063/1.4768806

Optically pumped lasing from a single pillar microcavity with InGaAs/GaAs quantum well potential fluctuation quantum dots

J. Appl. Phys. 105, 053513 (2009); 10.1063/1.3074364

Surface-emitting microlaser combining two-dimensional photonic crystal membrane and vertical Bragg mirror Appl. Phys. Lett. 88, 081113 (2006); 10.1063/1.2172730

Over $3 \mathrm{~W}$ high-efficiency vertical-external-cavity surface-emitting lasers and application as efficient fiber laser pump sources

Appl. Phys. Lett. 86, 211116 (2005); 10.1063/1.1935756

Vertically emitting annular Bragg lasers using polymer epitaxial transfer

Appl. Phys. Lett. 85, 3669 (2004); 10.1063/1.1807970

\section{A|P| $\left.\right|_{\text {Applied Physics }} ^{\text {Journal of }}$}

Journal of Applied Physics is pleased to announce André Anders as its new Editor-in-Chief 


\title{
Low dimensional GaAs/air vertical microcavity lasers
}

\author{
J. Gessler, ${ }^{1}$ T. Steinl, ${ }^{1}$ A. Mika, ${ }^{2}$ J. Fischer, ${ }^{1}$ G. Sęk, ${ }^{2}$ J. Misiewicz, ${ }^{2}$ S. Höfling, ${ }^{1, a)}$ \\ C. Schneider, ${ }^{1}$ and M. Kamp ${ }^{1}$ \\ ${ }^{1}$ Technische Physik, Physikalisches Institut and Wilhelm Conrad Röntgen-Research Center for Complex \\ Material Systems, Universität Würzburg, Am Hubland, D-97074 Würzburg, Germany \\ ${ }^{2}$ Institute of Physics, Wroclaw University of Technology, Wybrzeże Wyspiańskiego 27, 50-370 Wrocław, \\ Poland
}

(Received 22 January 2014; accepted 12 February 2014; published online 25 February 2014)

\begin{abstract}
We report on the fabrication of gallium arsenide (GaAs)/air distributed Bragg reflector microresonators with indium gallium arsenide quantum wells. The structures are studied via momentum resolved photoluminescence spectroscopy which allows us to investigate a pronounced optical mode quantization of the photonic dispersion. We can extract a length parameter from these quantized states whose upper limit can be connected to the lateral physical extension of the microcavity via analytical calculations. Laser emission from our microcavity under optical pumping is observed in power dependent investigations. (C) 2014 AIP Publishing LLC.
\end{abstract}

[http://dx.doi.org/10.1063/1.4866805]

GaAs microcavities based on distributed Bragg reflectors are key components in a large variety of photonic devices. ${ }^{1,2}$ The high reflectivity of dielectric mirrors facilitates low threshold lasing but also allows to confine light sufficiently long to observe quantum electrodynamical effects in semiconductor microcavities. ${ }^{3-5}$ As striking examples, strong light matter coupling in quantum well $(\mathrm{QW})$ or quantum dot (QD) microcavities based on distributed Bragg reflectors (DBRs) has been observed. ${ }^{4,6}$ Due to the small difference between the refractive indices of the routinely used lattice matched materials $\mathrm{AlAs}$ and GaAs (which is on the order of 0.5), a large number of dielectric mirror pairs satisfying the Bragg condition (typically 20-40) usually sandwich the optical cavity. This is necessary in order to realize microcavities with sufficiently high quality factors (Q-factors) to allow for the observation of pronounced lightmatter coupling effects or microcavity laser emission. An important parameter for the light-matter coupling is the optical mode volume, which depends on the light penetration depth into the mirrors, and therefore on the refractive index contrast. ${ }^{5}$ Several approaches to reduce the light penetration into the DBR mirrors have been reported on, including hybrid photonic crystal mirrors, ${ }^{7,8}$ plasmonic mirrors, ${ }^{9,10}$ selective oxidation of aluminum compound mirrors, ${ }^{11}$ and GaAs/air DBR mirrors. ${ }^{12}$ While plasmonic mirrors are especially appealing to dramatically reduce the effective cavity length, they inherently suffer from high absorption of near infrared photons and hence comparably low Q-factors. On the other hand, microcavities based on two hybrid photonic mirrors ${ }^{13}$ are, in particular, challenging to fabricate. The aimed approach in the work presented here is to develop a flexible DBR microcavity design with potentially ultra-high Q-factors on a double GaAs/air DBR microcavity with integrated quantum wells. In order to strongly reduce the effective cavity length, we maximize the difference in the refractive index of the alternating layers by selectively

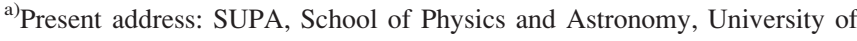
St. Andrews, St. Andrews KY16 9SS, United Kingdom.
}

removing the AlGaAs layers. The resulting low dimensional devices feature quality factors exceeding 3000 and show pronounced laser emission under optical pumping.

The epitaxially grown sample consists of a $\lambda$-cavity containing four $7 \mathrm{~nm}$ thick $\operatorname{In}_{0.13} \mathrm{Ga}_{0.87} \mathrm{As}$-QWs emitting at $1.388 \mathrm{eV}$. The QWs are separated by $4 \mathrm{~nm}$ thick GaAs barriers. The bottom DBR consists of $3.5 \mathrm{GaAs} / \mathrm{Al}_{0.7} \mathrm{Ga}_{0.3} \mathrm{As}$ mirror pairs while the top DBR is made of three mirror pairs. The layer thicknesses are designed for a photonic resonance at $1.363 \mathrm{eV}$ in the center of the wafer for the fully processed GaAs/air sample. The thickness of the $\mathrm{Al}_{0.7} \mathrm{Ga}_{0.3}$ As layers (which are selectively removed later on) is a quarter of the resonance wavelength in air while the GaAs $(n=3.614)$ layers fulfill a $3 \lambda / 4$ condition to gain more stability during the process and for the fully processed sample. In Fig. 1(a), a scanning electron microscope image of the grown structure is depicted.

After growth is completed, a $250 \mathrm{~nm}$ thick $\mathrm{SiO}_{2}$ layer is sputtered onto the wafer to serve as an etch mask. Then the sample is coated with PMMA (Polymethylmethacrylate) for electron beam lithography. The fabrication layout of our sample consists of pairs of holes (circles and rectangles) of different size and distance. The exposed resist is removed, and the two holes are etched with reactive ion etching through the $\mathrm{SiO}_{2}$. In a second etching step, the holes are etched deeply into the semiconductor. These holes serve as contact surface for the following selective wet etching step. $10 \%$ hydrofluoric acid is used as a chemical wet etch to remove the $\mathrm{Al}_{0.7} \mathrm{Ga}_{0.3}$ As layers and to realize a free standing area around each hole. Ideally, yet not necessarily, the complete distance between one pair of holes is free standing. A scanning electron microscopy image of such an underetched region can be seen in Fig. 1(b). To avoid a collapse during the drying process because of surface tension, t-Butyl alcohol sublimation ${ }^{14}$ is used. The resulting device formed by the free-standing GaAs/air membrane region in between the two holes is schematically sketched in Fig. 1(c).

Fig. 2(a) shows calculations of the mode intensities of a GaAs/air Bragg cavity and a standard AlAs/GaAs cavity. 

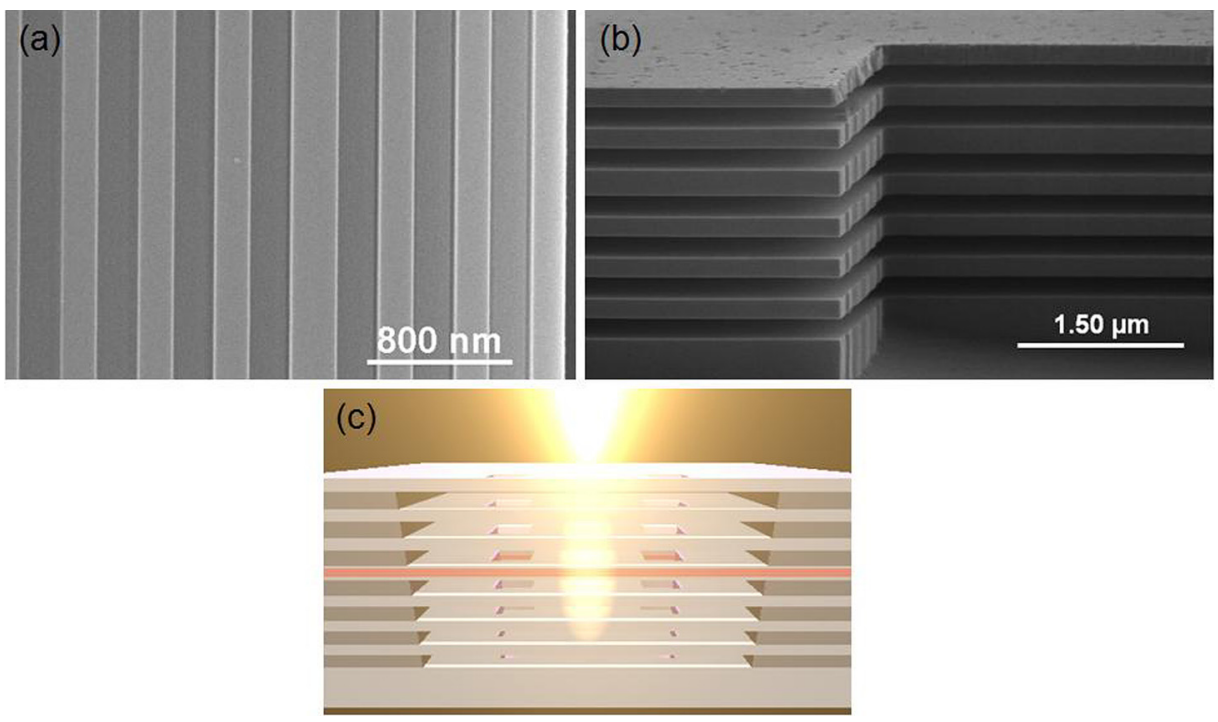

The intensity profiles were calculated by a plane wave expansion technique using the software CAMFR. ${ }^{15}$ Our simulations yield a reduction of the effective cavity length by a factor of $\sim 2$ in the GaAs/air approach. For instance, in the regime of strong light-matter coupling between quantum well excitons and cavity photons, such a reduction would directly lead to an increased Rabi-splitting by a factor of $\sqrt{2}$. Calculations of maximally expected Q-factors of ideal twodimensional microcavities obtained by transfer matrix calculations are presented in Fig. 2(b). As a result of the significantly higher contrast in the refractive index between the mirror materials, microcavities with theoretical Q-factors exceeding 100000 can be expected with only 3 GaAs/air mirror pairs (MPs). In contrast, $25 \mathrm{GaAs} / \mathrm{AlAs}$ MPs are at least necessary to theoretically design similarly cold cavity Q-factors.

In order to systematically study the photonic properties of our fabricated resonator samples, we have performed energy-momentum dispersion measurements. The Fourier space photoluminescence (PL) setup is composed of a standard micro-PL configuration with an additional far field lens to convert the real space image into the momentum-space $(\mathrm{k}-$ space) image. ${ }^{16}$ This setup allows a direct acquisition of the momentum-energy dispersion on a two-dimensional CCD screen.

We first discuss the influence of finite size effects on the emission spectra from our microcavity systems. The system size is set by the boundaries of the system: On one hand, this is the interface between the underetched area (air) and the as-grown area $\left(\mathrm{Al}_{0.7} \mathrm{Ga}_{0.3} \mathrm{As}\right)$; on the other hand the interface between the DBR to the deep etched holes defines a natural boundary. The latter is geometrically well defined. Its dimensions are given by the processing layout and the semiconductor to air transitions lead to a very deep optical confinement.

In Figs. 3(a) and 3(b), a representative energymomentum dispersion map is depicted. The sample was held at temperature of $70 \mathrm{~K}$ and excited with a $658 \mathrm{~nm}$ laser with a power of $35.8 \mathrm{~mW}$. A rich set of photonic resonances appear red detuned from the QW (emission energy: $1.390 \mathrm{eV}$ ) as discrete lines in energy. The low-dimensional character of the photonic resonances is reflected in their finite extension in k-direction together with their discrete character in energy.

Q-factors extracted from the ground state of the measured spectra surpass values of 1200 with maximum values of 3040 which are exemplarily depicted in Fig. 3(c). The spectrum shows a profile of the measurement taken in Fig. 3(a) around $\mathrm{k}_{\|}$equal zero. These values compare favorably to previous reports on GaAs based structures ${ }^{17}$ and InP based devices ${ }^{18}$ of similar design. We nevertheless note a strong deviation from the theoretical Q-factor which we attribute to the following reasons: we measure the Q-factor in the vicinity $(30 \mathrm{meV})$ of the QW emission. By the absorption of the $\mathrm{QW}$, the measured Q-factor is naturally reduced. ${ }^{19}$ On the (a)

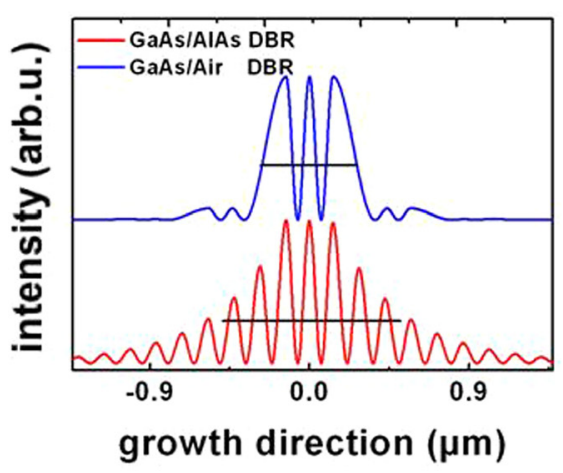

(b)

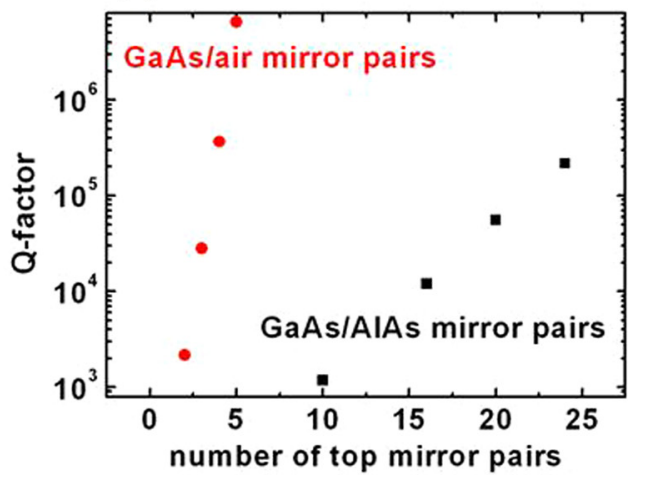

FIG. 2. (a) Comparison of the simulated mode intensities of a standard GaAs/AlAs DBR and the presented GaAs/air DBR design. The intensity mode profiles are normalized to one and shifted vertically for clarity and the black horizontal lines illustrate the effective cavity lengths. (b) Comparison of theoretical maximum Q-factor for planar samples. 
(a)

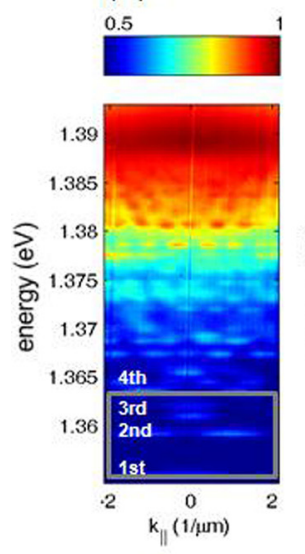

(c)

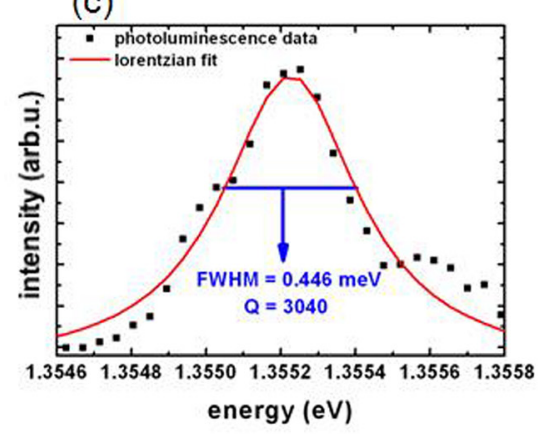

(b)

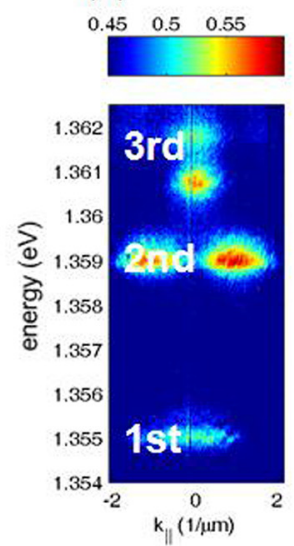

(d)

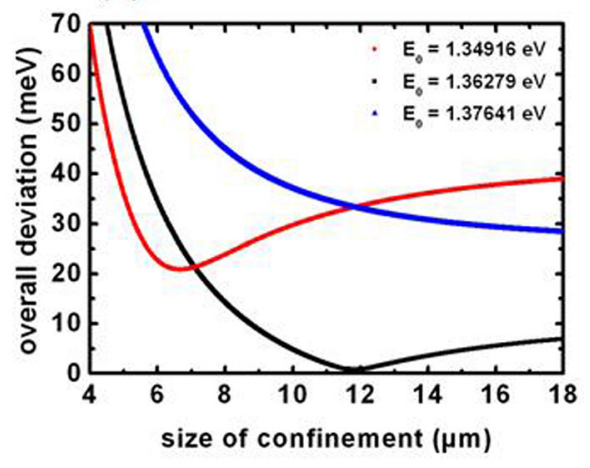

(e)

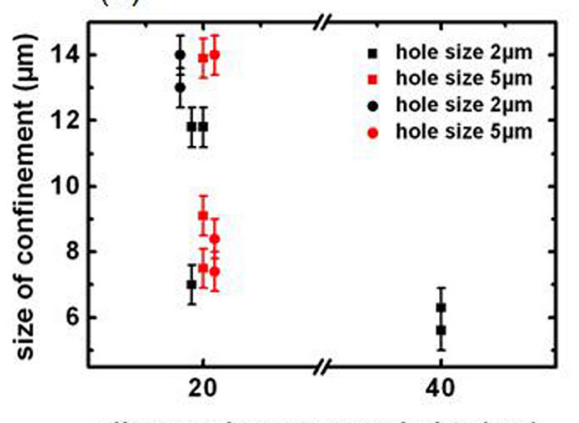

distance between two holes ( $\mu \mathrm{m})$
FIG. 3. (a) Photoluminescence spectrum with momentum-resolution. The image shows the dispersion recorded between two holes (distance, $20 \mu \mathrm{m}$; hole size, $2 \mu \mathrm{m}$; shape, rectangles). Indicated are the numbers of the states. (b) Zoom in the dispersion as indicated by the gray rectangle in Fig. 3(a). (c) Profile of the measurement taken in Fig. 3(a) around $\mathrm{k}_{\|}=0$. A Q-factor of 3040 was extracted from the linewidth of the resonance. (d) Result gained from 1D infinite potential well model. The deviation of the calculation from the measurement is plotted as a function of the lateral size of the confinement for three different energies of the photon ground state. (e) Result of several measurements: Circles and rectangles indicate the physical shape of the deeply etched pair of holes. The outcome can be divided into two groups: in group one, situated at $(7.3 \pm 1.1)$ $\mu \mathrm{m}$, the confinement is limited by the single under etched distance. The second group is located at $(13.1 \pm 1.0)$ $\mu \mathrm{m}$. The confinement is smaller than expected because of Anderson localization. contrary, we can exclude effects arising from the finite selectivity of HF. ${ }^{20}$ The expected difference in the layer thickness from the beginning of the deep dry etched holes to the center of the GaAs/air microcavity is below one atomic layer because of the high selectivity of hydrofluoric acid for AlAs. We also eliminate the influence of the mirror pairs sagging since the photonic mode does not shift along the connection line of the two deep dry etched holes within the resolution of our setup of $200 \mu \mathrm{eV}$.

We will now discuss the finite size effects leading to the observed mode quantization in Figs. 3(a) and 3(b). The photonic confinement in our system is generated by the physical boundaries in our system, such as the lateral interface from semiconductor to air (as sketched in Fig. 1(c)). The corresponding distance between two deep dry etched holes is specified by electron beam processing and amounts to either $20 \mu \mathrm{m}$ or $40 \mu \mathrm{m}$ in our study. Since the refractive index contrast between semiconductor and air is rather large, we assume that the optical mode is pinned to zero at this interface. In particular, for the $40 \mu \mathrm{m}$ spacing, it was not possible to realize a free-standing membrane. Optical microscopy images suggested a lateral size of the underetched region to $(10 \pm 3) \mu \mathrm{m}$, which is now limited by the transition from the highly reflective GaAs/air region to the GaAs/AlGaAs surrounding. To calculate the quantized mode energies, we use the analytical expression in, e.g., Ref. 21. The effective refractive index in our structure $(\sim 1.988)$ was estimated by averaging the refractive indices weighted by the field intensity in vertical direction.

Using this solid wall potential model, we can theoretically reproduce the experimental mode energies, using the ground state energy and the lateral size as free parameters. A measure for the excellence of the fit is the mean square deviation of the calculation from the measurement summed over all states. In Fig. 3(d), one can see the deviation as a function of the size of the confinement for three exemplarily selected ground state energies of the photon mode. A distinct minimum of the deviation, namely, $0.72 \mathrm{meV}$, yields a lateral confinement on the order of $11.8 \mu \mathrm{m}$.

Fig. 3(e) compiles the result of this analysis, which we carried out for various devices with different parameters (distance, shape, and size of the holes). Our results can be divided into two groups. One with the size of confinement located around $(13.1 \pm 1.0) \mu \mathrm{m}$, the second situated at (7.3 \pm 1.1$) \mu \mathrm{m}$ including the measurements taken from devices with $40 \mu \mathrm{m}$ distance of the holes. This suggests that confinement, in particular for large hole spacings, is generated by one deep dry etched hole on the one side and by the interface between the underetched and the as-grown area on the other side. For the $20 \mu \mathrm{m}$ distance of the holes, this suggests that a small wall of not underetched area is still remaining, which we could not detect via optical microscopy. The measurements with the larger size of confinement $(13 \mu \mathrm{m})$ emerge only at the $20 \mu \mathrm{m}$ distance of the holes, which nevertheless deviates from the expected value around $20 \mu \mathrm{m}$. We attribute this to Anderson localization ${ }^{22}$ originating from disorder in the etched edge of the deep holes, leading to an additional effective photonic confinement. We note that we neglected the dependency of the refractive index on the wavelength of the bulk material during the calculation of the length parameter. In our case, this reduces the effective refractive index for higher quantized modes leading to a slightly underestimated length parameter. Another consequence visible in Fig. 3(e) is the shape of the deep dry etched holes (circles 


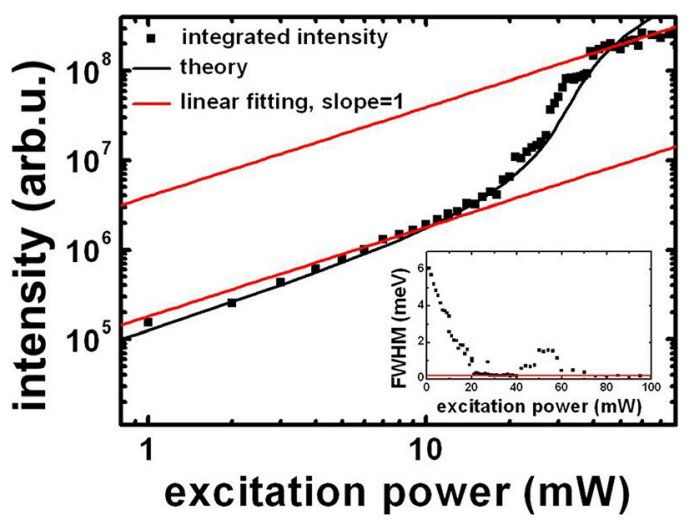

FIG. 4. Integrated photoluminescence intensity vs. the excitation power in a double logarithmic presentation. The straight lines result from a fitting procedure in the appropriate data range (red) and the fit of a rate equation model (black). The s-shape is an indicator for laser operation. The inset shows the linewidth decreasing to the resolution limit with increasing excitation power. The rise around $55 \mathrm{~mW}$ is attributed to mode competition with a second photonic mode.

and rectangles as indicated) which have no influence on the size of the confinement.

To reach the lasing regime in our sample, we excited it with a Titanium:sapphire laser at $839.1 \mathrm{~nm}$ wavelength in continuous wave mode and recorded the photoluminescence as a function of excitation power at a temperature of $70 \mathrm{~K}$. In the integrated intensity plot of the emission peak (Fig. 4), we can observe the lasing threshold for optical pumping at an excitation power of $20 \mathrm{~mW}$ with a decreasing linewidth down to the resolution of our setup of $200 \mu \mathrm{eV}$. The increase of the linewidth at an excitation power of $55 \mathrm{~mW}$ is attributed to a mode competition from a second photonic mode. ${ }^{23}$ The S-shape and the decreasing linewidth are typical for laser operation in the weak coupling regime. From theoretical calculations based on a rate equation model, ${ }^{24}$ we can extract the fraction of spontaneous emitted photons $\beta=(1.02 \pm 0.01) \%$ which are funneled into the cavity. A rough estimate of the excitation power we used to reach the lasing threshold is $1.6 \mathrm{~kW} / \mathrm{cm}^{2}$ which we extracted from the beam diameter $(4 \mu \mathrm{m})$ and the reflectivity of the top DBR $(>99 \%)$ at the used excitation wavelength. Over the whole excitation range, the emission shifts around $3.3 \mathrm{meV}$ towards the red, which indicates sample heating.

In conclusion, we have demonstrated the fabrication of high quality low dimensional GaAs/air microcavities. The photoluminescence spectra of our sample are dominated by discrete resonances, correlating with the finite size of our structures in three dimensions. Excitation power dependent investigations of our structures revealed a clear evidence of photon lasing, supported by a pronounced threshold behavior and a decreasing linewidth down to the resolution limit as a signature of temporal coherence. We anticipate that our device technology can be adapted for the realization of compact polariton lasers due to the comparably short physical cavity length and the capability to employ opto-mechanical tuning techniques.

This work was financially supported by the state of Bavaria and the bilateral project of the Deutsche Forschungsgemeinschaft (Project LIEPOLATE) and the Polish Ministry of Science and higher education (Project No. DPN/N99/DFG/2010). We thank J. Beetz, S. Brodbeck, R. Weih, and M. Amthor for fruitful discussions and technical support during sample preparation and measurement. Fruitful discussions with R. Ohta, S. Iwamoto, and Y. Arakawa are gratefully acknowledged.

${ }^{1}$ T. E. Sale, Vertical Cavity Surface Emitting Lasers (Research Studies Press, 1995).

${ }^{2}$ K. Iga, "Vertical-cavity surface-emitting laser: Its conception and evolution,” Jpn. J. Appl. Phys., Part 1 47, 1-10 (2008).

${ }^{3}$ J.-M. Gerard, Topics in Applied Physics, edited by P. Michler (2003), Vol. 90, pp. 315-347.

${ }^{4}$ C. Weisbuch, M. Nishioka, A. Ishikawa, and Y. Arakawa, Phys. Rev. Lett. 69(23), 3314 (1992).

${ }^{5}$ A. Kavokin and G. Malpuech, Cavity Polaritons (Elsevier Academic Press, 2003).

${ }^{6}$ J. P. Reithmaier, G. Sek, A. Löffler, C. Hofmann, S. Kuhn, S. Reitzenstein, L. V. Keldysh, V. D. Kulakovskii, T. L. Reinecke, and A. Forchel, Nature 432, 197-200 (2004).

${ }^{7}$ M. C. Huang, Y. Zhou, and C. J. Chang-Hasnain, Nat. Photonics 1(2), 119-122 (2007).

${ }^{8}$ B. Zhang, Z. Wang, S. Brodbeck, C. Schneider, M. Kamp, S. Höfling, and H. Deng, Light: Sci. Appl. 3, e135 (2014).

${ }^{9}$ O. Gazzano, S. Michaelis de Vasconcellos, K. Gauthron, C. Symonds, J. Bloch, P. Voisin, J. Bellessa, A. Lemaître, and P. Senellart, Phys. Rev. Lett. 107, 247402 (2011).

${ }^{10}$ M. Schubert, M. Grossmann, O. Ristow, M. Hettich, A. 1. Bruchhausen, E. C. S. Barretto, E. Scheer, V. Gusev, and T. Dekorsy, Appl. Phys. Lett. 101, 013108 (2012).

${ }^{11}$ M. Rattier, H. Benisty, R. P. Stanley, J. F. Carlin, R. Houdre, U. Oesterle, and T. F. Krauss, IEEE J. Sel. Top. Quantum Electron. 8(2), 238-247 (2002).

${ }^{12}$ C. Grossmann, C. Coulson, G. Christmann, I. Farrer, H. E. Beere, D. A. Ritchie, and J. J. Baumberg, Appl. Phys. Lett. 98, 231105 (2011).

${ }^{13}$ J. Li, D. Fattal, M. Fiorentino, and R. G. Beausoleil, Phys. Rev. Lett. 106(19), 193901 (2011).

${ }^{14}$ C.-J. Kim, J. Y. Kim, and B. Sridharan, Sens. Actuators, A 64, 17-26 (1998).

${ }^{15}$ P. Bienstman and R. Baets, Opt. Quantum Electron. 33, 327-341 (2001).

${ }^{16}$ C. W. Lai, N. Y. Kim, S. Utsunomiya, G. Roumpos, H. Deng, M. D. Fraser, T. Byrnes, P. Recher, N. Kumada, T. Fujisawa, and Y. Yamamoto, Nature 450, 529-532 (2007).

${ }^{17}$ H. Chen, Q. Mo, Z. Huang, O. Shchekin, C. Cao, S. Lipson, and D. G. Deppe, in Conference on Lasers and Electro-Optics, Surface Emitting Lasers (CWC), Baltimore, Maryland, 1 June 2003.

${ }^{18}$ H. Hillmer, J. Daleiden, C. Prott, F. Romer, S. Irmer, V. Rangelov, A. Tarraf, S. Schuler, and M. Strasser, Appl. Phys. Lett. B 75(1), 3-13 (2002).

${ }^{19}$ S. Reitzenstein, C. Hofmann, A. Gorbunov, M. Strauss, S. H. Kwon, C. Schneider, A. Löffler, S. Höfling, M. Kamp, and A. Forchel, Appl. Phys. Lett. 90, 251109 (2007).

${ }^{20}$ E. Yablonovitch, T. Gmitter, J. P. Harbison, and R. Bhat, Appl. Phys. Lett. 51, 2222 (1987).

${ }^{21}$ J. P. Reithmaier, M. Röhner, H. Zull, F. Schäfer, A. Forchel, P. A. Knipp, and T. L. Reinecke, Phys. Rev. Lett. 78, 378 (1997).

${ }^{22}$ P. W. Anderson, Phys. Rev. 109, 5 (1958).

${ }^{23}$ F. Albert, C. Hopfmann, S. Reitzenstein, C. Schneider, S. Höfling, L. Worschech, M. Kamp, W. Kinzel, A. Forchel, and I. Kanter, Nat. Commun. 2, 366 (2011).

${ }^{24}$ P. Jaffrennou, J. Claudon, M. Bazin, N. S. Malik, S. Reitzenstein, L. Worschech, M. Kamp, A. Forchel, and J. M. Gerard, Appl. Phys. Lett. 96, 071103 (2010). 